\title{
Experiencia inicial del cierre sin sutura de la gastrosquisis. Reporte de casos en un hospital de la ciudad de Puebla, México
}

\author{
Manuel Gil-Vargas* \\ Jaime Hernández-Hernández** \\ Mary Sol Saavedra-Pacheco***
}

\begin{abstract}
*Cirujano pediatra. Socio Numerario de la Academia Mexicana de Pediatría. Hospital General del Sur "Eduardo Vázquez N". Puebla. Puebla. México. **Residente de pediatría III año. Hospital General del Sur "Eduardo Vázquez N". Puebla. Puebla. México.

***Médico Pasante del Servicio Social. Facultad de Medicina. Benemérita Universidad Autónoma de Puebla. Puebla. Puebla. México.

Correspondencia: Dr. Manuel Gil Vargas. Dirección: 11 oriente 1828, Interior 503, Puebla, Puebla, México. Tel: +52 12221360456 . Correo electrónico: gilvm@yahoo.com
\end{abstract}

Resumen

Introducción: La gastrosquisis es una de las malformaciones congénitas más frecuentes. En países subdesarrollados su mortalidad es mayor al $15 \%$ siendo la sepsis su principal causa. Su manejo implica el cierre de la pared abdominal mediante diferentes métodos, dependiendo del nivel de complejidad y del tipo de intervención. Objetivo: Describir la experiencia inicial del cierre sin sutura de la gastrosquisis, en una unidad de neonatología de Puebla, México. Materiales y métodos: Se realizó un estudio observacional, descriptivo, transversal y retrospectivo; en el cual se estudiaron 9 expedientes de recién nacidos con cierre sin sutura de gastrosquisis, en el Hospital General del Sur de Puebla. Resultados: Se estudiaron a los pacientes de Diciembre 2018 a Agosto 2019, de los cuales el 56\% correspondía al sexo masculino. Se dividieron en 3 grupos: Cierre inmediatamente posterior a la cesárea (67\%), retardo inmediato (11\%), y retardo con uso del separador Alexis (22\%). De los pacientes, el promedio fue de $25.67 \pm 1.56$ días de estancia intrahospitalaria. Únicamente un paciente del grupo 2 presentó como complicación sepsis, el resto no manifestó complicación alguna. Conclusiones: El cierre sin sutura en nuestros pacientes resultó ser un procedimiento confiable y con buen pronóstico para su evolución, debido a resultados favorables en los días de estancia intrahospitalaria y el tiempo de inicio de la vía oral. MÉD.UIS.2020;33(3): 43-7.

Palabras clave: Gastrosquisis. Ventilación mecánica. Recién nacido. Sepsis.

\section{Initial experience of the sutureless closure of gastroschisis. Report of cases in a hospital in the city of Puebla, México}

\begin{abstract}
Introduction: Gastroschisis is one of the most frequent congenital malformations. In underdeveloped countries, its mortality is higher than $15 \%$, being sepsis the first cause. Its management consists on the closure of the abdominal wall by different methods, depending on its level of complexity and the type of intervention. Objective: Describe the initial experience of the sutureless closure of the gastroschisis, at a unit of neonatology in Puebla, Mexico. Materials and methods: A descriptive, transversal and retrospective study was performed to evaluate 9 medical charts of newborns with sutureless closure of gastroschisis, at the "Hospital General del Sur de Puebla" Results: The patients were studied from December 2018 to August 2019, of which 56\% were males. They were divided into 3 groups. With close immediately after the c-section (67\%), immediate delay (11\%) and delay using the Alexis retractor (22\%). The mean time of length hospital stay of the patients was $25.67 \pm 1.56$ days. The only one with a complication was the patient from group 2 that developed sepsis. Conclusions: The closure without suture in our patients turned out to be a reliable procedure with a good prognosis to their evolution, due to favorable results in the length of hospital stay and the timing of enteral feeding. MÉD.UIS.2020;33(3): 43-7.
\end{abstract}

Keywords: Gastroschisis. Sutureless surgical procedures. Case reports. Infant. Newborn.

Artículo recibido el 30 de mayo de 2020 y aceptado para publicación el 20 de octubre de 2020. 
¿Cómo citar este artículo?: Gil-Vargas M, Hernández-Hernández J, Saavedra-Pacheco MS. Experiencia inicial del cierre sin sutura de la gastrosquisis. Reporte de casos en un hospital de la ciudad de Puebla, México. MÉD.UIS.2020;33(3):43-7. doi: 10.18273/revmed.v33n3-2020005

\section{Introducción}

La gastrosquisis es una malformación congénita de la pared abdominal anterior detectable durante el periodo gestacional'. Actualmente su frecuencia mundial va en aumento, reportando una incidencia de $0.4 \%$ a $11.7 \%$ casos por cada 10000 recién nacidos; algunos autores reportan que se ha incrementado de forma rápida y mantenido hasta 10-20 veces en las últimas 3 décadas ${ }^{2,3}$. En México, del 2000 al 2014, la incidencia aumentó de 2.09 a 6.85 por cada 10,000 nacimientos. En países subdesarrollados su mortalidad es mayor al $15 \%$, por lo general consecuente a sepsis ${ }^{4}$.

La gastrosquisis se divide en compleja y no compleja. La compleja es cuando macroscópicamente hay perforación o atresia intestinal, y para ello el manejo quirúrgico que se recomienda es el uso de silo más cierre diferido (por etapas); en la no compleja está indicado el cierre sin sutura ${ }^{5}$. Para el manejo de la gastrosquisis no compleja existen diversas formas de cierre, que con el paso del tiempo se han ido modificando y mejorando. Se dividen en cierre primario o cierre diferido. El cierre primario es la reducción del intestino eviscerado a la cavidad abdominal, con síntesis de la fascia y piel, utilizando una técnica con o sin sutura. Las desventajas de realizar cierre con sutura son ingreso a quirófano, incisiones más grandes con deformidad de la pared abdominal y mayor tiempo de intubación endotraqueal. Cuando el contenido intestinal no puede entrar a la cavidad abdominal se realiza el cierre diferido, el cual consiste en el uso del silo (colocar una bolsa de silastic alrededor de los bordes del defecto de la pared abdominal y mediante plicaturas progresivas de la misma, introducir el contenido extraabdominal a la cavidad para así lograr el cierre de la fascia y piel en un segundo tiempo quirúrgico). También está el cierre sin sutura mediante el separador Alexis que permite con el paso de los días la introducción del contenido intestinal a la cavidad abdominal5 ${ }^{5-7}$.

En estudios internacionales refieren que la mortalidad de la gastrosquisis ha pasado del $95 \%$ a menos del $50 \%$ cuando se realiza la detección oportuna de la malformación de forma prenatal ${ }^{3}$. En varios países de América latina las malformaciones congénitas se encuentran dentro de las principales causas de morbilidad y mortalidad en la infancia, por lo que un diagnóstico temprano resulta de gran importancia para brindar un manejo oportuno de las mismas ${ }^{9}$ El cierre sin sutura de la gastrosquisis ofrece a los recién nacidos no pasar a quirófano ya que esta reducción se puede hacer dentro de la misma sala de partos o en la unidad de cuidados intensivos neonatales.

El objetivo del presente estudio es describir la experiencia inicial en los días de estancia intrahospitalaria, ventilación mecánica, inicio de la vía oral y las complicaciones en el cierre sin sutura de la gastrosquisis en una unidad de neonatología de un hospital de tercer nivel de Puebla, México.

\section{Materiales y métodos}

Se trató de un estudio observacional, descriptivo, transversal y retrospectivo que se llevó a cabo con base en una revisión de expedientes de neonatos, nacidos por cesárea, con diagnóstico de gastrosquisis quienes fueron sometidos a cierre sin sutura de la pared abdominal en el Hospital General de Puebla "Dr. Eduardo Vázquez N.", ya que anteriormente este tipo de pacientes pasaban a quirófano para su manejo. Se incluyeron todos los recién nacidos con gastrosquisis no compleja tratados quirúrgicamente completando su estancia intrahospitalaria en este centro hospitalario.

Los criterios de exclusión incluyeron a todos los recién nacidos con cierre sin sutura de la gastrosquisis que hayan sido tratados quirúrgicamente en otra unidad médica y a los que fallecieron antes del cierre sin sutura de la pared abdominal. Ninguno de los expedientes de los pacientes participantes se eliminó del proceso.

En este estudio de investigación se recopilaron los datos de Septiembre a Octubre de 2019 y fue aprobado por el comité de ética e investigación de la unidad hospitalaria.

Se analizaron las variables en una base de datos en el programa de estadística SPSS versión 25. Las 
variables discretas se expresaron como frecuencia y porcentaje, y para las variables continuas se expresaron como media y desviación estándar. Para comprobar si las variables se distribuyeron normalmente, se utilizó la prueba de ShapiroWilk y posteriormente la de homogeneidad. Se realizó análisis de varianza para diferencia de tres poblaciones. Se considerará estadísticamente significativo si el valor de P es inferior a 0.05.

El separador Alexis es un retractor de heridas que permite acceso a manos libres en el sitio de operación, aumenta al máximo la asistencia quirúrgica y proporciona la protección de la herida. Los órganos eviscerados se introdujeron en el separador Alexis y el anillo de este se colocó en la cavidad abdominal sin sutura para cerrar el defecto secuencialmente ${ }^{10}$.

\section{Resultados}

Se atendieron de diciembre 2018 a agosto 2019 a nueve recién nacidos con gastrosquisis de los cuales cinco fueron masculinos (56\%) y cuatro femeninos (44\%), los cuales se dividieron en tres grupos (Ver Tabla 1).

Tabla 1. Pacientes sometidos a cierre sin sutura.

\begin{tabular}{|l|l|l|l|}
\hline & $\begin{array}{l}\text { Cierre } \\
\text { inmediatamente } \\
\text { posterior a la } \\
\text { cesárea }\end{array}$ & $\begin{array}{l}\text { Retardo } \\
\text { inmediato }\end{array}$ & $\begin{array}{l}\text { Retardo } \\
\text { con uso de } \\
\text { separador } \\
\text { Alexis. }\end{array}$ \\
\hline Recién nacidos & 6 & 1 & 2 \\
\hline
\end{tabular}

Fuente: autores.

El paciente de cierre con retardo inmediato ingresó a nuestra institución a las 72 horas de vida extrauterina. Mientras que en los dos pacientes donde se utilizó el separador Alexis se redujo el contenido intestinal a la cavidad abdominal con una media de $4.5 \pm 2.5$ días de retardo (mínimo 2 días - máximo 7 días), con una desviación estándar de 3.5 días.

En cuanto a los días de estancia intrahospitalaria de los nueve recién nacidos, se obtuvo una media de $25.67 \pm 1.56$ días, mínima de 18 días y una máxima de 36 días con una desviación estándar de 5.56 con una $p=0.939$. (Ver Figura 1)

De los pacientes con cierre inmediato posterior a la cesárea se tuvo en promedio una estancia intrahospitalaria de 26.17 días, con un mínimo de
18 días y un máximo de 36 días con una desviación estándar de 6.2 días. Para el grupo 2 se tuvo una estancia intrahospitalaria de 24 días. Para el grupo 3 una media de 25, con mínimo de 20 y máximo de 30 días con una desviación estándar de 7.07 días.

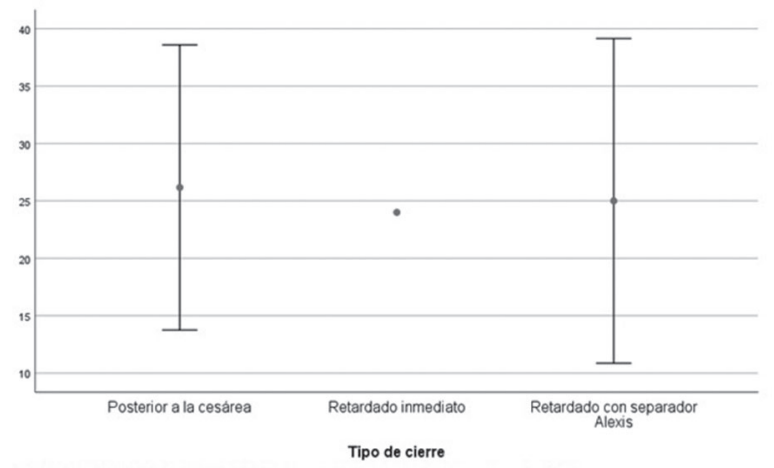

Figura 1. Figura 1. Media de los días de estancia intrahospitalaria grupos 1, 2 y 3.

Fuente: autores.

El uso de la ventilación mecánica para los nueve pacientes obtuvimos una media de $129.89 \pm 39.31$ horas, una mínima de 24 horas y una máxima de 355 horas, con una desviación estándar de 117.94 horas y una $p=0.02$.

Para los pacientes con cierre inmediato posterior a la cesárea correspondió una media de 56.5 horas, un mínimo de 24 horas y un máximo de 96 horas, con una desviación estándar de 28.04 horas.

El único paciente de cierre con retardo inmediato estuvo bajo ventilación mecánica por espacio de 262 horas. Para el grupo 3 fue una media de 293 horas, una mínima de 213 horas y una máxima de 355 horas, y una desviación estándar de 87.68 horas. (Ver Figura 2)

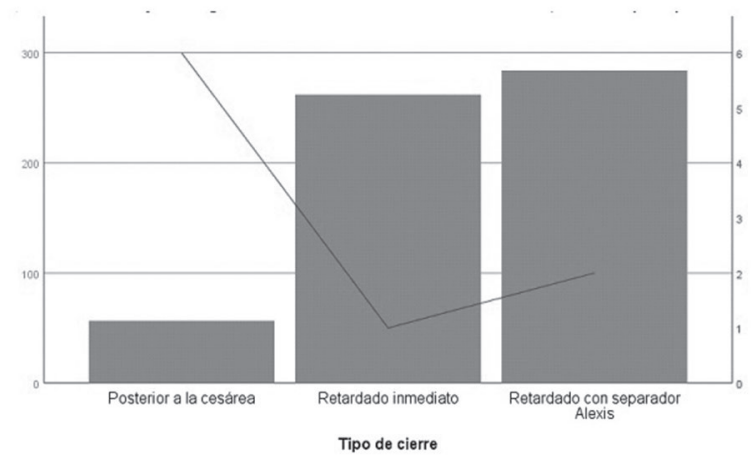

Figura 2. Media de las Horas de Ventilación Mecánica grupos 1, 2 y 3.

Fuente: autores. 
El inicio de la vía oral en general tuvo para los 9 pacientes una media de $269 \pm 41.71$ horas, una mínima de 52 horas y una máxima de 384 horas, con una desviación estándar de 125.15 horas, con una $\mathrm{p}=0.871$. El grupo 1 tuvo una media de 233 horas y el grupo 3 de 369.5 horas. (Ver Tabla 2)

Tabla 2. Inicio de la vía oral en cada grupo.

\begin{tabular}{|c|c|c|c|}
\hline $\mathbf{n}$ & Mínimo & Máximo & Media (DE) \\
\hline Grupo 1 & 52 & 384 & 139.05 \\
\hline Grupo 2 & 284 & 284 & 0 \\
\hline Grupo 3 & 355 & 384 & 20.5 \\
\hline
\end{tabular}

Fuente: autores.

El paciente con cierre retardado inmediato presentó como complicación sepsis manejada con antibioticoterapia de amplio espectro (cefotaxima y amikacina), evolucionando de forma satisfactoria. El resto no manifestó complicación alguna.

\section{Discusión}

Con el paso del tiempo el manejo quirúrgico de la gastrosquisis ha encontrado nuevas formas de cierre: desde el uso de silo, el cierre bajo anestesia y recientemente el cierre sin sutura ${ }^{11,12}$. La literatura menciona que una de las mejores técnicas es el cierre sin sutura, la cual tiene mejor pronóstico al eliminar el uso de anestesia general y el dar la opción de realizarse en la unidad de cuidados intensivos neonatal y no en quirófano; a diferencia del cierre primario con sutura, el cual, además, tiene mayor riesgo de desarrollar complicaciones como el síndrome compartimental; este tipo de cierre tiene un rango de éxito entre el 50 y el $83 \%$ dependiendo el estudio ${ }^{6}$, además de que estéticamente no hay cicatriz ni deformidad de la pared abdominal. La función intestinal se mantiene posterior al evento quirúrgico sin sutura, permitiendo administrar alimentación temprana disminuyendo el tiempo de estancia hospitalaria, pero se recomienda la ampliación de los estudios, para poder demostrar que las mejoras sean significativas ${ }^{13}$.

Los datos obtenidos en nuestro estudio no muestran una significación estadística con respecto a los días de estancia intrahospitalaria $(p=0.939)$. Sin embargo, en promedio son menos días (25.67) a comparación de lo reportado por Witt, Riboh y Bruzoni. Bruzoni con una muestra de 39 pacientes tuvo una media de 49.3 días de estancia intrahospitalaria, Witt tuvo una media de 36.7 días en una muestra de 97 pacientes y Riboh una media de 24.8 con 26 pacientes ${ }^{12,14,15}$.
El tiempo de ventilación mecánica en relación con lo descrito en la literatura fue mayor, una limitante fue el uso de sedación y relajación. El cierre sin sutura permite reducir la necesidad de ventilación mecánica"1. Se reportó una media de 129.89 horas contra 163.2 en los pacientes de Witt $^{8}$. Teniendo en cuenta a Ogasawara, nosotros tuvimos menos tiempo a los pacientes con ventilación mecánica, ya que en su estudio de siete pacientes en Japón, en promedio fueron 137.1 horas las que permanecieron los pacientes intubados ${ }^{16}$. Eggink concluyó en su estudio realizado en Texas, que los pacientes a los que se les realiza un cierre diferido se mantienen en ventilación mecánica por más tiempo ${ }^{17}$.

A pesar de que en nuestra serie los recién nacidos tuvieron sedación y relajación, el inicio de la vía oral se alcanzó en menor tiempo en comparación con los reportado en la literatura (269 horas contra 669.6, 403.2 y 1080 horas) $)^{12,14,15}$.

En un estudio realizado por Machuca ${ }^{18}$ el inicio de la vía oral en promedio fue a las 408 horas, en contraste con nuestro estudio donde se logró una media de 269 horas. Romay en promedio presentó el inicio de la vía oral a los 312 horas $^{19}$. Weinsheimer reporta que obtuvo significación estadística con una $p<0.4$ al iniciar la vía oral en 99 pacientes con gastrosquisis con cierre sin sutura, en un estudio realizado en Canadá ${ }^{20}$.

La sepsis es la causa más frecuente de morbilidad y mortalidad en el primer año de vida de estos pacientes ${ }^{21}$. En nuestro estudio únicamente se reportó en un paciente una complicación (sepsis), probablemente por el tamaño de la muestra o el tiempo de seguimiento posterior al cierre. En comparación, Schlueter afirma que en los primeros 60 días postquirúrgicos el $24 \%$ de sus pacientes tuvo alguna complicación: el 38\% sepsis y el 35\% infección de la herida quirúrgica ${ }^{22}$. Por otro lado, Tullie afirma que en su estudio el $86 \%$ de los pacientes sometidos a cierre sin sutura presentaron como complicación hernia umbilical en el cierre diferido. Hasta el momento no tenemos defectos umbilicales, sin embargo, se sugiere el seguimiento de estos niños hasta los cuatro años ${ }^{23}$.

Una de nuestras limitantes fue el pequeño tamaño de la muestra y el periodo de seguimiento aún es muy corto, sin embargo, no descartamos como complicación más frecuente a largo plazo hernias umbilicales. 


\section{Conclusiones}

Los días de estancia intrahospitalaria en este trabajo fueron menores a lo reportado en la literatura. El inicio de la vía oral se logró reiniciar de manera pronta, reduciendo las horas en comparación con lo citado en la literatura, y la única complicación presente fue sepsis en un solo paciente, una de las complicaciones de la intervención mencionadas en la literatura.

\section{Referencias bibliográficas}

1. O'Connell RV, Dotters-Katz SK, Kuller JA, Strauss RA. Gastroschisis: A Review of Management and Outcomes. Obstet Gynecol Surv. 2016;71(9): 537-544.

2. Rattan KN, Sonika P, Singh R, Yadav K, Hota D. Manual ward reduction of gastroschisis without anesthesia, a safe procedure - 8 years experience. J Ped Neonat Individual Med. 2017;6(1):1-5.

3. Nazer-Herrera J, Karachon-Essedin L, Cifuentes-Ovalle L, AssarCuevas R. Gastrosquisis: ¿una pandemia con tasas en aumento? Experiencia del estudio colaborativo latino americano de malformaciones congénitas. Rev Chil pediatría. 2016;87(5):380-6.

4. Zalles-Vidal C, Peñarrieta-Daher A, Bracho-Blanchet E, IbarraRios D, Dávila-Perez R, Villegas-Silva R, et al. A Gastroschisis bundle: effects of a quality improvement protocol on morbidity and mortality. J Pediatr Surg. 2018;53(11).

5. Petrosyan M, Sandler AD. Closure methods in gastroschisis. Semin Pediatr Surg. 2018;27(5):304-8.

6. Baeza-Herrera C, Cortés-García R, Cano-Salas M del C, GarcíaCabello LM, Martínez-Leo B. Gastrosquisis. Su tratamiento en un estudio comparativo. Acta Pediatr Mex. 2011;32(5):266-72.

7. Dore-Reyes M, Triana-Junco P, Barrena-Delfa S, Encinas JL, RomoMuñoz M, Vilanova-Sánchez A, et al. Primary versus secondary closure: ventilatory and nutritional differences in patients with gastrosquisis. Cir Padiatr. 2016;29(2):49-53.

8. Salinas-Torres VM, Salinas-Torres RA, Cerda-Flores RM, Martínez-de-Villarreal LE. Prevalence, Mortality, and Spatial Distribution of Gastroschisis in Mexico. J Pediatr Adolesc Gynecol. 2018;31(3):232-7.

9. Calderón-Alvarado AB, Rojas-Villegas M del S, Dehesa-López E. Prevalencia de malformaciones congénitas detectadas al nacimiento en un hospital de segundo nivel en Sinaloa. Acta pediatr Mex. 2017;38(6):363-80.

10. Protector W. Alexis ${ }^{\circledR}$ Alexis ${ }^{\circledR}$ Wound Protector / Retractor. 2017;2016-7

11. Grabski DF, Hu Y, Vavolizza RD, Rasmussen SK, Swanson JR, McGahren ED, et al. Sutureless closure: a versatile treatment for the diverse presentations of gastroschisis. J Perinatol. 2019;1(1):1-7.

12. Riboh J, Abrajano CT, Garber K, Hartman G, Butler MA, Albanese CT, et al. Outcomes of sutureless gastroschisis closure. J Pediatr Surg. 2009;44(10):1947-51.

13. Harris J, Poirier J, Selip D, Pillai S, N Shah A, Jackson CC, et al. Early Closure of Gastroschisis After Silo Placement Correlates with Earlier Enteral Feeding. J Neonatal Surg. 2015;4(3):28.

14. Witt RG, Zobel M, Padilla B, Lee H, MacKenzie TC, Vu L. Evaluation of Clinical Outcomes of Sutureless vs Sutured Closure Techniques in Gastroschisis Repair. JAMA Surg. 2019;154(1):3339.

15. Bruzoni M, Jaramillo JD, Dunlap JL, Abrajano C, Stack SW, Hintz SR, et al. Sutureless vs Sutured Gastroschisis Closure: A Prospective Randomized Controlled Trial. J Am Coll Surg. 2017:224(6):1091-96.

16. Ogasawara Y, Okazaki T, Kato Y, Lane GJ, Yamataka A. Spontaneous sutureless closure of the abdominal wall defect in gastroschisis using a commercial wound retractor system. Pediatr Surg Int. 2009;25(11):973-6.

17. Eggink B, Richardson CJ, Malloy MH, Angel CA. A 20-year case review of infants with gastroschisis born in Galveston, Texas. J Pediatr Surg. 2006;41(6):1103-8.

18. Machuca AJ, Guido O, Fernández LA, Cordero G, Yllescas E, Carrera S. Gastrosquisis: resultados en una institución de tercer nivel. Perinatol y Reprod Humana. 2017:31(2):68-72.

19. Lacarrubba J, Genes L, Céspedes E, Mendieta E, Rivarola C, Rovira A, et al. Gastrosquisis. Experiencia en los últimos 30 meses. Pediatria (Asunción). 2013;40(3): 217-225.

20. Weinsheimer RL, Yanchar NL, Bouchard SB, Kim PK, Laberge J-M, Skarsgard ED, et al. Gastroschisis closure-does method really matter? J Pediatr Surg. 2008;43(5):874-8.

21. Bello ABR, Ruiz PS, de la Fuente EA, Badillo CC, Montes JM. Defectos del cierre de la pared abdominal: gastrosquisis. Prog. Obstet. Ginecol. 2011;54(12):612-7.

22. Schlueter RK, Azarow KS, Hines AG, Varman M, Abdessalam SF, Raynor SC, et al. Identifying strategies to decrease infectious complications of gastroschisis repair. J Pediatr Surg. 2015;50(1):98-101.

23. Tullie LGC, Bough GM, Shalaby A, Kiely EM, Curry JI, Pierro A, et al. Umbilical hernia following gastroschisis closure: a common event? Pediatr Surg Int. 2016;32(8): 811-4. 